\title{
Pharmacokinetics of ertapenem in patients with multidrug-resistant tuberculosis
}

\author{
Sander P. van Rijn ${ }^{1,7}$, Richard van Altena ${ }^{2,7}$, Onno W. Akkerman², \\ Dick van Soolingen ${ }^{3,4}$, Tridia van der Laan ${ }^{4}$, Wiel C.M. de Lange ${ }^{2}$, \\ Jos G.W. Kosterink ${ }^{1,5}$, Tjip S. van der Werf ${ }^{6}$ and Jan-Willem C. Alffenaar ${ }^{1}$
}

Affiliations: ${ }^{1}$ University of Groningen, University Medical Center Groningen, Dept of Clinical Pharmacy, Groningen, The Netherlands. ${ }^{2}$ University of Groningen, University Medical Center Groningen, Tuberculosis Center Beatrixoord, Haren, The Netherlands. ${ }^{3}$ Radboud University Nijmegen Medical Center, Depts of Pulmonary Diseases and Medical Microbiology, Nijmegen, The Netherlands. ${ }^{4}$ National Tuberculosis Reference Laboratory, National Institute for Public Health and the Environment (RIVM), Bilthoven, The Netherlands. ${ }^{5}$ University of Groningen, University Medical Center Groningen, Section Pharmacotherapy and Pharmaceutical Care, Dept of Pharmacy, Groningen, The Netherlands. 'University of Groningen, University Medical Center Groningen, Depts of Internal Medicine and Pulmonary Diseases and Tuberculosis, Groningen, The Netherlands. ${ }^{7}$ Both authors contributed equally.

Correspondence: Jan-Willem C. Alffenaar, University of Groningen, University Medical Center Groningen, Dept of Hospital and Clinical Pharmacy, P.0. Box 30.001, 9700 RB Groningen, The Netherlands.

E-mail: j.w.c.alffenaardumcg.nl

ABSTRACT Treatment of multidrug-resistant (MDR) and extensively drug-resistant (XDR) tuberculosis (TB) is becoming more challenging because of increased levels of drug resistance against second-line TB drugs. One promising group of antimicrobial drugs is carbapenems. Ertapenem is an attractive carbapenem for the treatment of MDR- and XDR-TB because its relatively long half-life enables once-daily dosing.

A retrospective study was performed for all patients with suspected MDR-TB at the Tuberculosis Center Beatrixoord of the University Medical Center Groningen (Haren, the Netherlands) who received ertapenem as part of their treatment regimen between December 1, 2010 and March 1, 2013. Safety and pharmacokinetics were evaluated.

18 patients were treated with $1000 \mathrm{mg}$ ertapenem for a mean (range) of 77 (5-210) days. Sputum smear and culture were converted in all patients. Drug exposure was evaluated in 12 patients. The mean (range) area under the concentration-time curve up to $24 \mathrm{~h}$ was $544.9(309-1130) \mathrm{h} \cdot \mathrm{mg} \cdot \mathrm{L}^{-1}$. The mean (range) maximum observed plasma concentration was $127.5(73.9-277.9) \mathrm{mg} \cdot \mathrm{L}^{-1}$.

In general, ertapenem treatment was well tolerated during MDR-TB treatment and showed a favourable pharmacokinetic/pharmacodynamic profile in MDR-TB patients. We conclude that ertapenem is a highly promising drug for the treatment of MDR-TB that warrants further investigation.

@ERSpublications

Pharmacokinetic and safety data show ertapenem to be a promising drug for MDR-TB treatment http://ow.ly/V69DD

Received: Oct 062015 | Accepted after revision: Nov 15 2015 | First published online: Jan 072016

Conflict of interest: Disclosures can be found alongside the online version of this article at erj.ersjournals.com

Copyright OERS 2016 


\section{Introduction}

Treatment of multidrug-resistant (MDR) and extensively drug-resistant tuberculosis (TB) is becoming more challenging because of increased levels of drug resistance against second-line TB drugs. New drugs are being evaluated in clinical trials, but only bedaquiline and delamanid have entered the market to date. Therefore, antimicrobial drugs, which have been developed and labelled for other bacterial infections, may be of potential use in the treatment of MDR-TB.

One promising group of antimicrobial drugs is carbapenems [1,2]. An early in vitro experiment showed that imipenem and meropenem were active against Mycobacterium tuberculosis [3]. ChAmBers et al. [4] showed that imipenem has antimycobacterial activity in mice and humans. Imipenem and meropenem are currently listed as group 5 drugs for the treatment of MDR-TB [5]. More recently, clinical experience of carbapenems in MDR-TB patients showed promising results [6, 7].

Carbapenems are poor substrates for $\beta$-lactamase $\mathrm{C}(\mathrm{BLaC})$ due to rapid acylation and slow deacylation. Therefore, unlike $\beta$-lactams, they are not rapidly hydrolysed by BLaC and thus maintain their potential activity against $M$. tuberculosis [8]. The binding of carbapenems to L,D-transpeptidases results in inhibition of the peptidoglycan polymerisation of the cell wall [9]. Combined with a $\beta$-lactamase inhibitor, such as clavulanate, activity against M. tuberculosis is higher [10].

Efficacy of carbapenems is correlated with the percentage of time the free plasma drug concentration transcends the minimum inhibitory concentration (MIC) (tfree>MIC). Maximal bactericidal activity is reached if the $t$ free $>$ MIC is $\geqslant 40 \%$ of the dosing interval $[11,12]$. To reach this target for Gram-positive, Gram-negative and anaerobic bacterial infections, ertapenem is given intravenously at a dose of $1000 \mathrm{mg}$ once daily [13]. Ertapenem has the advantage over other carbapenems of a long half-life of $4 \mathrm{~h}$, enabling once-daily dosing [12], which is attractive for MDR-TB treatment. Another advantage is that ertapenem is not affected by drugdrug interactions as it is neither metabolised by cytochrome P450 nor a substrate for P-glycoprotein [14].

To include ertapenem among the other carbapenems as a group 5 drug for the treatment of MDR-TB, additional pharmacokinetic and safety data are urgently needed [15]. Therefore, the objective of this study was to evaluate pharmacokinetics and safety in patients that received ertapenem as part of their treatment regimen for MDR-TB.

\section{Patients and methods}

\section{Patients}

All patients suspected of MDR-TB at the Tuberculosis Center Beatrixoord of the University Medical Center Groningen (Haren, the Netherlands) who received ertapenem as part of their treatment regimen between December 1, 2010 and March 1, 2013 were included in this retrospective study. The study was evaluated by the Medical Ethical Review Board of the University Medical Center Groningen (METC 2013-492). The need for written informed consent was waived for the retrospective collection and analysis of anonymous data because it was not required under Dutch law (Medical Research Involving Human Subjects Act (WMO)). For each MDR-TB suspect, the following data were collected: age, sex, weight, height, ethnicity, drug susceptibility pattern, localisation of TB, antiretroviral therapy, sputum conversion, adverse effects (AEs) induced by ertapenem, dose, total exposure to ertapenem and duration of treatment.

\section{Drug susceptibility to ertapenem}

Drug susceptibility testing of ertapenem was performed with and without clavulanic acid using the Middlebrook 7H10 agar dilution method at the Dutch National Tuberculosis Reference Laboratory (National Institute for Public Health and the Environment (RIVM), Bilthoven, the Netherlands) [16].

\section{Pharmacokinetics and pharmacodynamics}

All patients received ertapenem at a dosage of $1000 \mathrm{mg}$ once daily, given as i.v. infusion in $30 \mathrm{~min}$. In all MDR-TB patients, routine plasma concentrations were collected at steady state to assess drug exposure and thus enable individualised dosing. For plasma sampling, a peripheral i.v. catheter was inserted. Patency of the peripheral catheter was maintained by a saline drip. Before a blood sample was taken, the drip was stopped and the first $4 \mathrm{~mL}$ of blood were discarded. The samples were collected before administration and at $1,2,3,4,5,6,8$ and $12 \mathrm{~h}$ post dosage and stored at $-80^{\circ} \mathrm{C}$ until analysis. Plasma concentrations were assessed and validated using a validated liquid chromatography-tandem mass spectrometry method in the Laboratory of Clinical Toxicology and Drugs Analysis of the Dept of Clinical Pharmacy and Pharmacology at the University Medical Center Groningen (Groningen, the Netherlands) [17]. Population pharmacokinetic parameters were calculated using the KinPOP module. Both KinFIT and KinPOP were part of the software package MWPharm 3.82 (Mediware Information Systems B.V., Eindhoven, the Netherlands). The tfree $>$ MIC was calculated as this has been proposed to be the best pharmacokinetic/ pharmacodynamic parameter to predict in vivo efficacy of carbapenems [10]. The free drug concentration 
was assumed to be 5\% [12,18]. European Committee on Antimicrobial Susceptibility Testing (EUCAST) MIC values for ertapenem (non-species related) of 0.5 and $1.0 \mathrm{mg} \cdot \mathrm{L}^{-1}$ were used to calculate tfree>MIC.

\section{Safety and tolerability}

Reported AEs in medical charts were used to evaluate the safety of ertapenem. Specific attention was paid to AEs mentioned in earlier studies, i.e. diarrhoea and vomiting. The Naranjo algorithm was used to evaluate for causality between AEs that occurred and ertapenem [19].

\section{Statistics and pharmacokinetic evaluation}

SPSS 20 was used as statistical software (SPSS, Virginia, IL, USA). Correlations between pharmacokinetic parameters and patient characteristics were analysed using the Spearman correlation coefficient. MIC data were statistically analysed using a methodology for censored MIC data [20].

\section{Results}

\section{Patients}

18 patients treated with ertapenem, mean (range) age 29 (13-66) years, were retrieved. Ertapenem was part of the treatment regimen because of suspected extensive drug resistance, intolerance to second-line drugs or a combination of both. Based on the results of the drug susceptibility test, ertapenem was discontinued in three patients who appeared to have drug-susceptible TB. There was an unequal sex distribution as eight $(44.4 \%)$ patients were male and $10(55.5 \%)$ patients were female. The mean (range) body mass index was $21.3(13.7-32.6) \mathrm{kg} \cdot \mathrm{m}^{-2}$. Patients originated predominantly from Africa (11 out of 18) and Europe (five out of 18). Patients were primarily diagnosed with pulmonary TB (13 out of 18); extrapulmonary sites were involved in seven patients.

The prescribed dosage of ertapenem was $1000 \mathrm{mg}$ once daily in all patients. The mean (range) total treatment duration of ertapenem was 77 (5-210) days. Drug resistance patterns of the patients to anti-TB agents are shown in table 1 . The most prescribed anti-TB drugs in the 18 patients were: moxifloxacin (17 patients), injectable (16 patients), linezolid (15 patients), clofazimine (eight patients), clarithromycin (six patients) and pyrazinamide (five patients).

In total, 15 patients completed treatment and were cured. Three patients were lost to follow-up. All patients with positive sputum smear converted within a mean (range) period of 17 (0-97) days. Cultures remained negative after culture conversion and no relapse of MDR-TB was observed.

\begin{tabular}{lcc} 
TABLE 1 Drug resistance patterns of the 18 study patients & & \\
Drugs by group & Resistant & Sensitive \\
\hline Group 1: first-line oral drugs & & \\
$\quad$ Isoniazid & $17(94.4)$ & $1(5.55)$ \\
$\quad$ Rifampicin & $15(83.3)$ & $3(16.6)$ \\
$\quad$ Pyrazinamide & $8(44.4)$ & $8(44.4)$ \\
$\quad$ Ethambutol & $11(61.1)$ & $6(33.3)$ \\
$\quad$ Rifabutin & $13(72.2)$ & $4(22.2)$ \\
Group 2: injectable agents & $14(77.7)$ & $4(22.2)$ \\
$\quad$ Streptomycin & $3(16.6)$ & $14(77.7)$ \\
$\quad$ Amikacin & $3(16.6)$ & $14(77.7)$ \\
$\quad$ Kanamycin & $5(27.7)$ & $12(66.6)$ \\
$\quad$ Capreomycin & & $15(83.3)$ \\
Group 3: fluoroquinolones & $2(11.1)$ & $10(55.5)$ \\
$\quad$ Moxifloxacin & & \\
Group 4: oral bacteriostatic second-line agents & $7(38.8)$ & $17(94.4)$ \\
$\quad$ Protionamide & & $7(38.8)$ \\
Group 5: agents with unclear role in treatment of drug-resistant TB & & $8(44.4)$ \\
$\quad$ Linezolid & & $18(100)$ \\
$\quad$ Clarithromycin & &
\end{tabular}

Data are presented as n (\%). TB: tuberculosis. " : groups defined by the World Health Organization. 


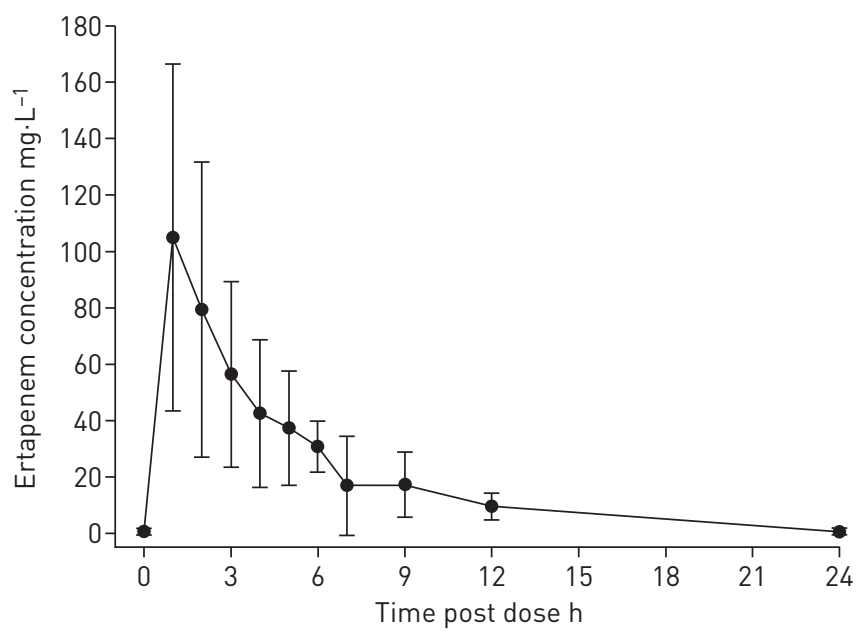

FIGURE 1 Ertapenem plasma concentration-time curves in 12 patients.

Drug susceptibility of M. tuberculosis to ertapenem

All the M. tuberculosis strains appeared susceptible to ertapenem. However, actual determination of the MIC was complicated by the fact that ertapenem itself appeared an instable compound at $37^{\circ} \mathrm{C}$ [17]. This was confirmed by the fact that after 7 days MIC values were lower than after 14 days. In addition, freshly prepared plates showed lower MIC values compared with plates stored at $4^{\circ} \mathrm{C}$. The refrigerator-stored plates showed lower MIC values than plates stored at room temperature. If ertapenem was combined with clavulanic acid, all MIC values were even lower.

\section{Pharmacokinetics and pharmacodynamics}

The plasma concentration-time curves were obtained in 12 patients with MDR-TB. In the remaining six patients, routine plasma concentrations were collected at a time-point at which they did not yet receive ertapenem or this drug was no longer administered. Three patients had multiple plasma concentrationtime curves and these were consistent. The mean curve is shown in figure 1. The mean (range) area under the concentration-time curve up to $24 \mathrm{~h}(\mathrm{AUC} 0-24)$ was $544.9(309-1130) \mathrm{h} \cdot \mathrm{mg} \cdot \mathrm{L}^{-1}$. The steady state pharmacokinetic parameters are shown in table 2. Based on a MIC of $0.25 \mathrm{mg} \cdot \mathrm{L}^{-1}, 11$ out of 12 patients exceeded a minimum of $40 \%$ tree $>$ MIC. In nine patients the MDR-TB remained susceptible with a MIC of $0.5 \mathrm{mg} \cdot \mathrm{L}^{-1}$. Only two patients exceeded a minimum of $40 \%$ tree $>$ MIC with a MIC of $1 \mathrm{mg} \cdot \mathrm{L}^{-1}$. The pharmacokinetic population model (KinPOP) of ertapenem showed a mean (range) clearance of 2.26 $(0.86-3.19) \mathrm{L} \cdot \mathrm{h}^{-1}$ per $1.73 \mathrm{~m}^{2}$ and a mean (range) volume of distribution of $8.79(4.76-13.57) \mathrm{L}$.

\section{Safety and tolerability}

In general, ertapenem was tolerated very well. In three patients, treatment with ertapenem was stopped. One of these patients suffered from Crohn's disease and developed MDR-TB after multiple dosages of infliximab, a tumour necrosis factor- $\alpha$ blocker $[21,22]$. This patient experienced allergic fever, shortly after administration of ertapenem and ethambutol. After reintroduction this happened again (Naranjo score $=4$ ). Both AEs subsided after withdrawal of the offending drug. In the second patient, ertapenem was stopped after an increase in liver enzymes (aspartate aminotransferase: $109 \mathrm{U} \cdot \mathrm{L}^{-1}$; alanine aminotransferase: $255 \mathrm{U} \cdot \mathrm{L}^{-1}$ ) after 13 days of treatment with ertapenem. However, after 2 months, while this patient was still on treatment without ertapenem, liver enzymes remained elevated (Naranjo score $=1$ ). In the third patient, kanamycin, linezolid and ertapenem were stopped due to line sepsis. This was considered not to be related to ertapenem. After removal of the venous access port, the patient recovered. Ertapenem and a new venous access port were not reintroduced, since they were not indicated anymore, due to low bacillary load at that time, and these i.v. drugs could be substituted with oral antimycobacterial drugs. None of the patients experienced diarrhoea, vomiting or dizziness.

TABLE 2 Pharmacokinetic parameters of ertapenem

\begin{tabular}{|c|c|c|c|c|c|}
\hline Study & $A U C_{0-24} \mathrm{~h} \cdot \mathrm{mg} \cdot \mathrm{L}^{-1}$ & $\mathrm{C}_{\max } \mathrm{mg} \cdot \mathrm{L}^{-1}$ & Half-life $h$ & Volume of distribution $\mathrm{L}$ & Clearance $L \cdot h^{-1}$ \\
\hline $1 \mathrm{~g}$ i.v. in MDR-TB patients & 544.9 (309-1130) & $127.5(73.9-277.9)$ & $2.4(2.047-3.528)$ & $7.3(2.612-11.1)$ & $2.1(0.0884-3.231)$ \\
\hline $1 \mathrm{~g}$ i.v. in healthy volunteers [18] & $572.1(572-672)$ & $154.9(145-175)$ & $4(3.8-4.4)$ & 8.2 & 1.8 \\
\hline
\end{tabular}

Data are presented as mean (range) and were calculated using KinFIT. AUC0-24: area under the concentration-time curve up to $24 \mathrm{~h}$; $\mathrm{C}_{\text {max: }}$ maximum observed plasma concentration; MDR-TB: multidrug-resistant tuberculosis. 


\section{Discussion}

This is the first study, following a clinical report of ertapenem [6], presenting pharmacokinetic and safety data in patients with MDR-TB. In comparison with healthy volunteers [18], MDR-TB patients showed lower AUC0-24 values. The mean volume of distribution calculated by KinFIT was lower for MDR-TB patients than for the healthy volunteers, whereas the mean clearance was higher for the MDR-TB patients than for the healthy volunteers. Our observations are consistent with other studies that showed a lower drug exposure of ertapenem in patients with infectious diseases [23-25]. More surprising was the intervariability in AUC between patients with MDR-TB. Other antimycobacterial drugs also show high variability and lower drug exposure in TB patients $[2,26,27]$. It is not yet completely clear why drug exposure is lower in TB patients. Apparently, stage of disease and altered body composition may potentially help to explain this observation.

Since ertapenem belongs to the class of $\beta$-lactams, ertapenem has a time-dependent bactericidal activity. The tfree $>$ MIC is therefore important to evaluate the efficacy of ertapenem against $M$. tuberculosis. NiCOLAU [11] indicated that, in the case of meropenem, with $40 \%$ tree $>$ MIC, bactericidal activity is observed, whereas $20 \%$ tfree $>$ MIC appeared to have bacteriostatic activity. Other studies have mentioned this tfree $>$ MIC as well [12, 14]. Protein binding of ertapenem was assumed to be 5\%, since ertapenem shows concentration-dependent plasma protein binding. Healthy volunteers, who average a peak plasma concentration of $150 \mathrm{mg} \cdot \mathrm{L}^{-1}$ after the end of infusion of $1 \mathrm{~g}$ of ertapenem, have a percentage of $8 \%$ unbound protein. When total drug plasma concentration declines below $50 \mathrm{mg} \cdot \mathrm{L}^{-1}$ peak plasma concentration, the percentage of unbound ertapenem is $\sim 5 \%$ [18]. It is very promising to notice that the non-species-related breakpoint of ertapenem of $0.5 \mathrm{mg} \cdot \mathrm{L}^{-1}$ was exceeded for $>40 \%$ of the day in the majority of patients, assuming that patients have a protein binding of $5 \%$. At a higher MIC value of $1 \mathrm{mg} \cdot \mathrm{L}^{-1}$, bacteriostatic activity could be expected. Therefore, ertapenem seems a very attractive drug for MDR-TB treatment. It seems warranted that doses of ertapenem higher than $1 \mathrm{~g} \cdot \mathrm{day}^{-1}$ should be used in the treatment of MDR-TB. However, in vitro experiments evaluating pharmacokinetic/pharmacodynamic targets for ertapenem against $M$. tuberculosis have yet to be performed. The hollow fibre infection model is suitable for pharmacokinetic/pharmacodynamic experiments and has already been used successfully [28].

Besides pharmacokinetics of ertapenem in patients with MDR-TB, additional safety data are described for the first time as well. Only one patient, with Crohn's disease, experienced AEs that might be potentially related to ertapenem. One can speculate that this may be related to an infusion-related AE, due to a developed immune disorder and eventually a consequence of an infliximab treatment. Drug-induced fever is a common AE of infliximab in the treatment of Crohn's disease [29]. AEs of other carbapenems, such as diarrhoea, nausea, vomiting, headache and rash, are well documented and found to be mild [1,30]. According to the product leaflet, ertapenem is given for a maximum of two consecutive weeks. Previous studies explored the safety and tolerability of ertapenem for this period of time and concluded that adverse side-effects were mild to moderate [13]. In our study we showed that AEs did not increase during prolonged treatment.

The measurement of actual MIC values was complicated by the fact that ertapenem is an instable compound at $37^{\circ} \mathrm{C}$. As drug susceptibility testing for $M$. tuberculosis takes $\geqslant 2$ weeks at $37^{\circ} \mathrm{C}$, it is highly likely that the initial drug concentration decreases rapidly over time. Unfortunately, with the current drug susceptibility systems (e.g. Mycobacterium growth indicator tube), this problem cannot be overcome, because the drug concentration in the medium cannot be corrected for a decrease in concentration due to degradation of the drug. Recently, the hollow fibre infection model solved this problem, as drug concentrations can be increased to correct for degradation [31]. However, these systems are expensive and difficult to manage, so it is not likely that routine drug susceptibility testing will be performed using hollow fibre systems. Another alternative may be the use of E-tests [32]. These are much cheaper and easier to employ, but it is unclear if they can help to overcome the instability of ertapenem. The most important limitations of our study are the retrospective character, the limited sample size and the absence of a control group, which prevent a meaningful conclusion on the efficacy of ertapenem. A further limitation is the inability to define MIC for ertapenem in clinical isolates. Nevertheless, all patients were cured and no relapse was noticed after being treated with the combination regimen including ertapenem. It is probable that the combination of drugs contributed to sputum culture conversion and favourable treatment outcome. This is in line with recently published data on tolerability and outcomes in five patients receiving ertapenem [6].

A recent editorial has proposed a new classification of anti-TB drugs. It marked the potential of carbapenems as group 5 drugs; however, carbapenems are still in need of proper evaluation and clinical evidence [33]. Before ertapenem can be labelled as a group 5 drug and used as part of MDR-TB treatment, a valid procedure to test drug susceptibility has to be made available. Ideally, the use of ertapenem would be supported by the results of a clinical trial.

In conclusion, this study provides new knowledge on the use of ertapenem in patients with MDR-TB, presenting pharmacokinetic and additional safety data. 


\section{References}

1 De Lorenzo S, Alffenaar JW, Sotgiu G, et al. Efficacy and safety of meropenem-clavulanate added to linezolid-containing regimens in the treatment of MDR-/XDR-TB. Eur Respir J 2013; 41: 1386-1392.

2 Pranger $\mathrm{AD}$, van Altena R, Aarnoutse RE, et al. Evaluation of moxifloxacin for the treatment of tuberculosis: 3 years of experience. Eur Respir J 2011; 38: 888-894.

3 Watt B, Edwards JR, Rayner A, et al. In vitro activity of meropenem and imipenem against mycobacteria: development of a daily antibiotic dosing schedule. Tuber Lung Dis 1992; 73: 134-136.

4 Chambers HF, Turner J, Schecter GF, et al. Imipenem for treatment of tuberculosis in mice and humans. Antimicrob Agents Chemother 2005; 49: 2816-2821.

5 Caminero JA, Sotgiu G, Zumla A, et al. Best drug treatment for multidrug-resistant and extensively drug-resistant tuberculosis. Lancet Infect Dis 2010; 10: 621-629.

6 Tiberi S, D'Ambrosio L, De Lorenzo S, et al. Ertapenem in the treatment of multidrug-resistant tuberculosis: first clinical experience. Eur Respir J 2016; 47: 333-336.

7 Winters N, Butler-Laporte G, Menzies D. Efficacy and safety of World Health Organization group 5 drugs for multidrug-resistant tuberculosis treatment. Eur Respir J 2015; 46: 1461-1470.

8 Hugonnet JE, Blanchard JS. Irreversible inhibition of the Mycobacterium tuberculosis $\beta$-lactamase by clavulanate. Biochemistry 2007; 46: 11998-12004.

9 Cordillot M, Dubée V, Triboulet S, et al. In vitro cross-linking of Mycobacterium tuberculosis peptidoglycan by L,D-transpeptidases and inactivation of these enzymes by carbapenems. Antimicrob Agents Chemother 2013; 57: 5940-5945.

10 Veziris N, Truffot C, Mainardi JL, et al. Activity of carbapenems combined with clavulanate against murine tuberculosis. Antimicrob Agents Chemother 2011; 55: 2597-2600.

11 Nicolau DP. Pharmacokinetic and pharmacodynamic properties of meropenem. Clin Infect Dis 2008; 47: Suppl. 1, S32-S40.

12 Zhanel GG, Wiebe R, Dilay L, et al. Comparative review of the carbapenems. Drugs 2007; 67: 1027-1052.

13 Teppler H, Gesser RM, Friedland IR, et al. Safety and tolerability of ertapenem. J Antimicrob Chemother 2004; 53: Suppl. 2, ii75-ii81.

14 Nix DE, Majumdar AK, DiNubile MJ. Pharmacokinetics and pharmacodynamics of ertapenem: an overview for clinicians. J Antimicrob Chemother 2004; 53: Suppl. 2, ii23-ii28.

15 Dooley KE, Obuku EA, Durakovic N, et al. World Health Organization group 5 drugs for the treatment of drug-resistant tuberculosis: unclear efficacy or untapped potential? J Infect Dis 2013; 207: 1352-1358.

16 van Klingeren B, Dessens-Kroon M, van der Laan T, et al. Drug susceptibility testing of Mycobacterium tuberculosis complex by use of a high-throughput, reproducible, absolute concentration method. J Clin Microbiol 2007; 45: 2662-2668.

17 van Rijn SP, Wessels AM, Greijdanus B, et al. Quantification and validation of ertapenem using a liquid chromatography-tandem mass spectrometry method. Antimicrob Agents Chemother 2014; 58: 3481-3484.

18 Majumdar AK, Musson DG, Birk KL, et al. Pharmacokinetics of ertapenem in healthy young volunteers. Antimicrob Agents Chemother 2002; 46: 3506-3511.

19 Naranjo CA, Busto U, Sellers EM, et al. A method for estimating the probability of adverse drug reactions. Clin Pharmacol Ther 1981; 30: 239-245.

20 van de Kassteele J, van Santen-Verheuvel MG, Koedijk FD, et al. New statistical technique for analyzing MIC-based susceptibility data. Antimicrob Agents Chemother 2012; 56: 1557-1563.

21 Cantini F, Niccoli L, Goletti D. Adalimumab, etanercept, infliximab, and the risk of tuberculosis: data from clinical trials, national registries, and postmarketing surveillance. J Rheumatol Suppl 2014; 91: 47-55.

22 Bumbacea D, Arend SM, Eyuboglu F, et al. The risk of tuberculosis in transplant candidates and recipients: a TBNET consensus statement. Eur Respir J 2012; 40: 990-1013.

23 Brink AJ, Richards GA, Schillack V, et al. Pharmacokinetics of once-daily dosing of ertapenem in critically ill patients with severe sepsis. Int J Antimicrob Agents 2009; 33: 432-436.

24 Burkhardt O, Derendorf H, Welte T. Ertapenem: the new carbapenem 5 years after first FDA licensing for clinical practice. Expert Opin Pharmacother 2007; 8: 237-256.

25 Sauermann R, Burian B, Burian A, et al. Tissue pharmacokinetics of ertapenem at steady-state in diabetic patients with leg infections. J Antimicrob Chemother 2013; 68: 895-899.

26 Alsaad N, van Altena R, Pranger AD, et al. Evaluation of co-trimoxazole in the treatment of multidrug-resistant tuberculosis. Eur Respir J 2013; 42: 504-512.

27 Dong $\mathrm{H}$, Wang X, Dong Y, et al. Clinical pharmacokinetic/pharmacodynamic profile of linezolid in severely ill intensive care unit patients. Int J Antimicrob Agents 2011; 38: 296-300.

28 Gumbo T, Louie A, Deziel MR, et al. Selection of a moxifloxacin dose that suppresses drug resistance in Mycobacterium tuberculosis, by use of an in vitro pharmacodynamic infection model and mathematical modeling. J Infect Dis 2004; 190: 1642-1651.

29 Hess S, Hospach T, Nossal R, et al. Life-threatening disseminated tuberculosis as a complication of TNF- $\alpha$ blockade in an adolescent. Eur J Pediatr 2011; 170: 1337-1342.

30 Zhanel GG, Simor AE, Vercaigne L, et al. Imipenem and meropenem: comparison of in vitro activity, pharmacokinetics, clinical trials and adverse effects. Can J Infect Dis 1998; 9: 215-228.

31 Srivastava S, van Rijn SP, Sherman C, et al. Sterilizing activity of ertapenem (ETP) against Mycobacterium tuberculosis (Mtb). Poster presented at Interscience Conference of Antimicrobial Agents and Chemotherapy (ICAAC), San Diego, CA, USA, September 17-21, 2015.

32 Esteban J, Ortiz A, Jiménez MS. Usefulness of E-test strips for testing susceptibility of Mycobacterium tuberculosis complex strains. Eur J Clin Microbiol Infect Dis 2005; 24: 856-857.

33 Caminero JA, Scardigli A. Classification of antituberculosis drugs: a new proposal based on the most recent evidence. Eur Respir J 2015; 46: 887-893. 